\title{
Hyper-Rayleigh scattering of gold nanorods and their relationship with linear assemblies of gold nanospheres
}

\author{
Jérôme Nappa, ${ }^{a}$ Guillaume Revillod, ${ }^{a}$ Jean-Pierre Abid, ${ }^{b}$ Isabelle Russier-Antoine, ${ }^{a}$ \\ Christian Jonin, ${ }^{a}$ Emmanuel Benichou, ${ }^{a}$ Hubert H. Girault ${ }^{b}$ and Pierre F. Brevet* ${ }^{a}$ \\ a Laboratoire de Spectrométrie Ionique et Moléculaire, UMR CNRS 5579, Université \\ Claude Bernard Lyon 1, Bâtiment Alfred Kastler, 43 Boulevard du 11 Novembre 1918, \\ 69622 Villeurbanne cedex, France.E-mail: pfbrevet@lasim.univ-lyon1.fr; \\ Fax: +33 (0)472 4458 73; Tel: +33 (0)4724458 73 \\ ${ }^{b}$ Laboratoire d'Electrochimie Physique et Analytique, Ecole Polytechnique Fédérale de \\ Lausanne, Ecublens, CH-1015 Lausanne, Switzerland
}

Received 25th March 2003, Accepted 11th April 2003

First published as an Advance Article on the web 27th October 2003

The surface plasmon enhanced hyper-Rayleigh scattering light collected from an aqueous solution of gold nanorods is reported. A non negligible part of the signal is attributed to a photoluminescence background attributed to the electron-hole recombination following multiphoton excitation of $\mathrm{d}$-valence band electrons into the sp-conduction band. This radiative relaxation process is likely favored by the presence of the organic species adsorbed at the surface of the nanorods. The absolute value for the hyperpolarisability of nanorods is also compared by the external reference method to that of para-nitroaniline and found to be rather large although an absolute value cannot be given because the exact number density of the gold nanorods is unaccessible. This value is however compared with values reported for linear assemblies of gold spherical nanoparticles and further support the simple model of gold metal ellipsoids to describe the hyper-Rayleigh light intensities. The polarisation analysis of the hyper-Rayleigh scattering light is also determined for gold nanorods and compared to the expected one for gold nanospheres. For the latter spheres, the weakness of the signal intensities precludes a definite comparison with the model. On the opposite, for the nanorods, the polarisation dependence of the hyper-Rayleigh scattered light clearly deviates from the one expected for nanospheres.

\section{Introduction}

The electronic properties of metallic nanoparticles have received a considerable interest in the recent years owing to their potential applications in electronic and opto-electronic devices. ${ }^{1}$ The origin of this interest mainly lies in the collective excitation of the conduction band electrons of the particle, known as the surface plasmon (SP) resonance, the frequency of which lies within the visible optical spectrum for gold and silver spherical particles, about $2.36 \mathrm{eV}$ and $3.13 \mathrm{eV}$ in aqueous solutions respectively. As a result, optical techniques have been methods of choice to investigate these properties and recently nonlinear optical techniques like hyper-Rayleigh scattering (HRS) have proven to be very sensitive to SP resonances. ${ }^{2-4}$ Previous studies have mainly been

DOI: $10.1039 / \mathrm{b} 303308 \mathrm{~b}$

Faraday Discuss., 2004, 125, 145-156

145

This journal is (C) The Royal Society of Chemistry 2003 
devoted to the measurement of the absolute values of the hyperpolarisability of silver and gold metallic nanoparticles. It has in particular been noticed that these values where rather large considering the electric dipole forbidden origin of the nonlinear response.

Indeed, although full theoretical accounts of the microscopic origin of the hyper-Rayleigh light scattered off spherical nanoparticles are still needed, it has quickly been recognised that owing to the size of these small particles and their spherical shape, the source to the nonlinear polarisation is of electric quadrupole origin. ${ }^{5}$ In a recent work, Dadap et al. have described in greater details the different contributions to the HRS signal, using a pure surface nonlinear polarisation. ${ }^{6}$ To the lowest order of the expansion in $a / \lambda$ where $a$ is the nanoparticle radius and $\lambda$ the fundamental wavelength, the first contribution to the total HRS intensity is a non-locally excited electric dipole oscillating at the harmonic frequency whereas the second contribution can be recast in the form of a locally excited electric quadrupole. Experimental confirmation of this description has been brought in by Hao et al. ${ }^{7}$ For silver nanoparticles with a diameter of $32 \mathrm{~nm}$, the separation between the two contributions to the HRS intensity has been obtained using an angle integrated detection of the HRS light. The separation of the two contributions in this experiment relies on the large size of the nanoparticles, this separation being not achieved on gold nanoparticles with a diameter of $11 \mathrm{~nm}$, as shown in a recent set of experiments performed in our group. ${ }^{8}$ It is indeed known that the weight of higher order multipoles will rise with the increasing size of the nanoparticles. Furthermore the use of silver instead of gold enables sharper resonances because the interband transitions occur at higher energies than the energy of the SP resonance in silver.

Besides the fundamental problem of the proper description of the nonlinear optical response from spherical particles, assemblies of nanoparticles have also received attention since it has been demonstrated that large local field enhancements can appear in metal-dielectric films near the percolation threshold. ${ }^{9}$ As a result, nonlinear optical techniques like second harmonic generation, since they probe higher powers of the electromagnetic field as compared to linear techniques, should be sensitive methods to investigate the nonlinear optical response from arrangements of nanoparticles. ${ }^{10}$ In a previous work, a first step in this direction was performed by recording the HRS intensity from a collection of assemblies of spherical gold nanospheres. ${ }^{11}$ The aggregation of gold nanospheres into large aggregates with fractal geometries is indeed known to be induced by addition of pyridine to the particle solution. ${ }^{12}$ The initial step of this aggregation reaction is the formation of aggregates with a linear geometry as has been shown by TEM imaging. ${ }^{13}$ The HRS signal collected from the solution of gold linear aggregates was enhanced by a factor of about ten as compared to the HRS signal from the monodispersed solution. For low concentrations of pyridine, in a regime dominated by these linear aggregates, the HRS intensity could be correctly described with a simple model of ellipsoidal gold particles.

Therefore, to further support these studies aiming at better understanding the origin of the HRS signal from small linear aggregates of gold nanoparticles, we report the investigation of the HRS signal arising from a solution of gold nanorods. The geometry of these nanoparticles is indeed similar to the one expected for linear aggregates, with the short axis of the rod roughly equal to the radius of the aggregating nanoparticles and the long axis equal to the number of particles into the aggregate. We focus in this work on the absolute HRS intensity of the gold nanorod solution and the depolarisation factor of the HRS light, comparing these data to the corresponding data available for gold nanospheres.

\section{Experimental}

\section{Chemicals}

Tetrachloroauric acid $\left(\mathrm{HAuCl}_{4} \cdot 2 \mathrm{H}_{2} \mathrm{O}\right)$ and sodium borohydrate $\left(\mathrm{NaBH}_{4}\right)$ were purchased from Aldrich, trisodium citrate dihydrate from Fluka, L-ascorbic acid from Riedel-de Haën and $N$-cetyl$N, N, N$-trimethylammonium bromide (CTAB) from Merck. Millipore ultra-pure water was used in the synthesis and all chemicals were used as received.

All glassware was thoroughly cleaned with aqua regia prior to the preparation. The gold nanorods were prepared according to the litterature. ${ }^{14}$ The first step consists in the preparation of gold spherical nanoparticles with an average diameter of about $3.5 \pm 0.5 \mathrm{~nm}$ as determined by TEM imaging. This preparation was performed starting from a $20 \mathrm{~mL}$ aqueous solution of 
tetrachloroauric acid, corresponding to a concentration of $2.510^{-4} \mathrm{M}$, vigorously stirred in an icecold bath. $600 \mu \mathrm{L}$ of $0.1 \mathrm{M}$ aqueous solution of sodium borohydride was then slowly added. The solution was stirred for an another $30 \mathrm{~min}$. This solution was then used as the seeding solution for the growth of the nanorods.

In this second step, three beakers were prepared with $9 \mathrm{~mL}$ of the $2.510^{-4} \mathrm{M}$ tetrachloroauric acid aqueous solution, in the presence of $0.1 \mathrm{M}$ of aqueous CTAB. In each beaker, $50 \mu \mathrm{L}$ of an aqueous $0.1 \mathrm{M} \mathrm{L}$-ascorbic acid solution was added. Then, in the first beaker, $1 \mathrm{~mL}$ of the seeding $3.5 \mathrm{~nm}$ diameter particle solution was added. After $1 \mathrm{~min}, 1 \mathrm{~mL}$ of the first beaker was taken and introduced in the second beaker. After 30 seconds, $1 \mathrm{~mL}$ of this second beaker was then introduced into the third beaker. This third final solution was then left to settle for $2 \mathrm{~h}$ before centrifugation at $2500 \mathrm{rpm}$ during $12 \mathrm{~min}$. The precipitate was then redissolved in $10 \mathrm{~mL}$ of pure water. This final centrifugation step followed by redissolution was repeated up to six times to remove unreacted L-ascorbic acid or other excess organic compounds. The final solution was then used as prepared in the optical experiments.

The gold nanosphere solution was either purchased (Sigma, gold colloids, $20 \mathrm{~nm}$ diameter) or prepared following the method of Turkevich et al. ${ }^{15} 3.5 \mathrm{~mL}$ of a $1 \%$ sodium citrate solution was added to a boiling solution of $100 \mathrm{~mL}$ of $0.01 \%$ tetrachloroauric acid under constant stirring. Heating was continued for half an hour followed by slow cooling to room temperature. The preparation was performed under reflux at all time to maintain a constant volume. Transmission electron microscopy (TEM) photographs of the nanoparticle solution were taken and the size distribution determined from 200 particles was found to retain an average diameter of $11 \mathrm{~nm}$ with a standard deviation of about $10 \%$.

\section{Characterisation}

Transmission Electron Microscopy (TEM) imaging of the nanorods was obtained by placing a droplet of the colloidal solution onto a carbon copper grid dried in a glove box under nitrogen flow for $30 \mathrm{~min}$. The TEM micrographs were taken on a transmission electron Microscope (Philips, model CM20) operating at $200 \mathrm{keV}$. UV-visible absorption spectroscopy was performed on a compact spectrophotometer operating with a deuterium-halogen lamp (Ocean Optics, model SD1000).

\section{Non linear optical set-up}

The experimental HRS set-up was based on a femtosecond Ti:sapphire laser with a repetition rate of $76 \mathrm{MHz}$ delivering 150 femtosecond pulses with an average power in the range $50-100 \mathrm{~mW}$ at the operating wavelength of $990-1030 \mathrm{~nm}$, i.e. $1.20-1.25 \mathrm{eV}$ (Coherent, model Mira 900). The laser beam was passed through a half-wave plate to rotate the input polarization angle and a filter to remove any unwanted second-harmonic light. The laser beam was then focussed into the quartz spectrophotometric cell through a microscope objective and the HRS light collected at right angle through a $4 \mathrm{~cm}$ focal length lens and a polarizer. The light was then passed through a monochromator and detected by a cooled photomultiplier feeding a photon counter. The data were acquired over repeated runs with long integration times to enhance as much as possible the signalto-noise ratio and improve the count statistics. To record the noise level at all times, the laser beam was chopped and the signal gated with gated photon counting techniques. All data were then reported as the difference of the two signals acquired. Furthermore, the measurements were always performed at the exact harmonic frequency, corresponding to twice the fundamental energy, and at the slightly higher energy in order to measure the background signal arising from the photoluminescence of the nanoparticles, see below. Finally, the transmission for the horizontally and the vertically polarised light of the experimental set-up was carefully determined using an unpolarised $\mathrm{CW} \mathrm{He}-\mathrm{Ne}$ laser operating at $543 \mathrm{~nm}$ and the fluorescence of a sulforhodamine solution. The latter solution yields a fluorescence depolarisation ratio equal to unity since the rotational diffusion time of the sulforhodamine molecules is about $150 \mathrm{ps}$ while the fluorescence lifetime is a few nanoseconds. All the data were corrected for this observed bias. 


\section{Results and discussion}

\section{Linear optics}

From TEM imaging spectroscopy of the nanorod solution, it was found that the solution was a distribution of nanorods and nanospheres. This underlines the difficulty to fully separate the two distributions through the centrifugation process. The UV-visible absorption spectrum of the nanorod solution is displayed in Fig. 1 and confirms this composition. Two bands are clearly visible corresponding to the two non-degenerated surface plasmon (SP) resonances. For nanorods, the SP resonance band at high energy, namely $2.32 \mathrm{eV}$, corresponds to the transverse SP resonance along the short axis of the nanorod whereas the SP resonance at $1.88 \mathrm{eV}$ corresponds to the longitudinal SP resonance of the nanorod. The energy of the transverse SP resonance is also identical to the single three-fold degenerated SP resonance of a nanosphere.

The extinction coefficient of a solution of randomly oriented gold nanorods is given by: ${ }^{16,17}$

$$
\sigma=\frac{2 \pi N V \varepsilon_{\mathrm{m}}^{1 / 2}}{3 \lambda} \sum_{i} \frac{\varepsilon_{2} / P_{i}^{2}}{\left(\varepsilon_{1}+\left(1-P_{i}\right) \varepsilon_{\mathrm{m}} / P_{i}\right)^{2}+\varepsilon_{2}^{2}}
$$

where $N$ is the number of particles in the sample solution, $V=4 \pi a^{2} b$ the volume of the rod, $\varepsilon_{\mathrm{m}}$ the dielectric constant of the medium and $\varepsilon=\varepsilon_{1}+\mathrm{i} \varepsilon_{2}$ the complex dielectric constant of gold metal. The three parameters $P_{i}$ are the three depolarisation factors corresponding to the three axes of the nanorod defined as:

$$
P_{1}=\frac{1-e^{2}}{e^{2}}\left[\frac{1}{2 e} \ln \left(\frac{1+e}{1-e}\right)-1\right]
$$

with $P_{2}=P_{3}=\left(1-P_{1}\right) / 2$. The eccentricity $e$ of the nanorod is related of the aspect ratio $r=a / b$ through:

$$
e=\sqrt{1-\frac{1}{r^{2}}}
$$

Using the bulk gold metal dielectric constants ${ }^{18}$ the extinction coefficient spectrum as calculated from eqns. (1)-(3) is observed to be in disagreement with the experimental spectrum, in particular the calculated longitudinal mode has a much larger oscillator strength, see Fig. 1. The calculated spectrum is obtained for an aspect ratio of $r=3.2$ allowing an energy for the longitudinal mode identical to the experimental one. A calculated spectrum of a gold nanosphere is also shown for

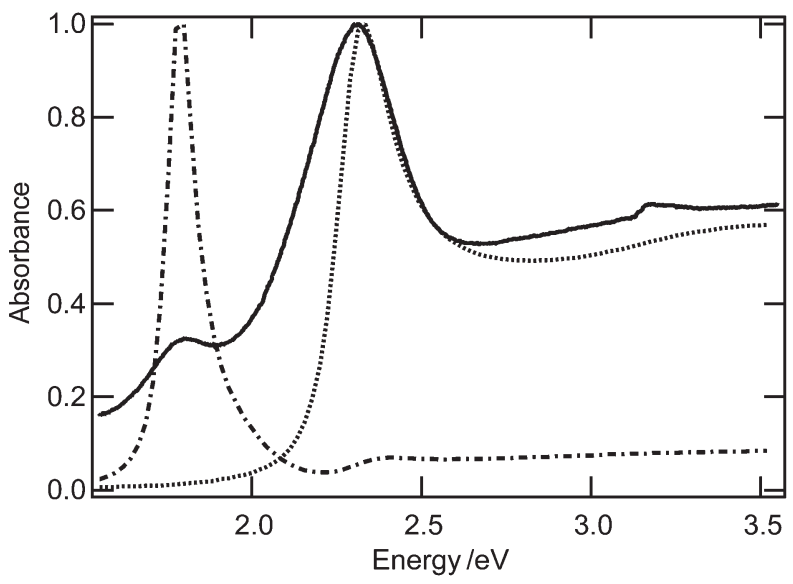

Fig. 1 UV-visible absorption spectrum of the gold nanorod solution (thick solid line). Also plotted are the calculated absorption spectrum for gold nanospheres (dotted line) and gold nanorods with an aspect ratio $r=3.23$ (dash-dotted line). 
comparison and the two compared to the experimental spectrum. The short axis of the nanorod is taken identical to the radius of the nanosphere. The ratio of the SP resonance bands for the longitudinal and the transverse modes clearly favors the transverse mode in the experimental spectrum while the reverse is true for the calculated one. This discrepancy between the two spectra is actually avoided if, in agreement with the TEM pictures, the solution is considered as a mixture of nanorods and nanospheres. Furthermore, it is expected that a distribution of nanorods with different aspect ratio has been synthesised. As a consequence, the solution is a mixture of particles with different shapes: nanospheres and nanorods with an aspect ratio ranging from unity to large values. On TEM pictures, several specimens with an aspect ratio close to 10 have been observed and although their weight in the total distribution is very weak because of the centrifugation process, their weight in the extinction coefficient spectrum scales with their volume. From the weights of the absorption spectra of the nanospheres and the nanorods required to match the experimental spectrum, it appears that the solution is composed of about $77 \%$ of nanospheres and $23 \%$ of nanorods with an aspect ratio of 3.2. A refinement of the calculated spectrum would be obtained by introducing a distribution of nanorods with different aspect ratios. Nanospheres and nanorods have also rather large radii or short and long axes therefore necessitating the incorporation of multipolar contributions to the their extinction coefficient spectrum. This is clearly shown on the red side of the transverse SP resonance band which is much broader than the calculated spectrum. Part of this broadening arises from the distribution of particles with different eccentricities, not separated during the centrifugation procedure as performed or with completely different shapes like cubes and prisms, such shapes having been observed during the TEM imaging characterisation procedure.

\section{Photoluminescence}

The monochromaticity of the second harmonic light generated from the mixed solution of nanospheres and nanorods was controlled first. This experimental procedure is necessary to ensure that the process is indeed the conversion of two photons at the fundamental frequency into one photon at the harmonic frequency. This experiment was performed by irradiating the sample cell at a fixed energy, namely $1.25 \mathrm{eV}$, and scanning the monochromator through a range of energies wider than the HRS band energy, see Fig. 2. The HRS band resulting from the two-photon conversion process appears at the expected energy of $2.50 \mathrm{eV}$ but a non vanishing background is recorded underneath. This background signal accounts for about $30 \%$ of the total signal intensity over the whole

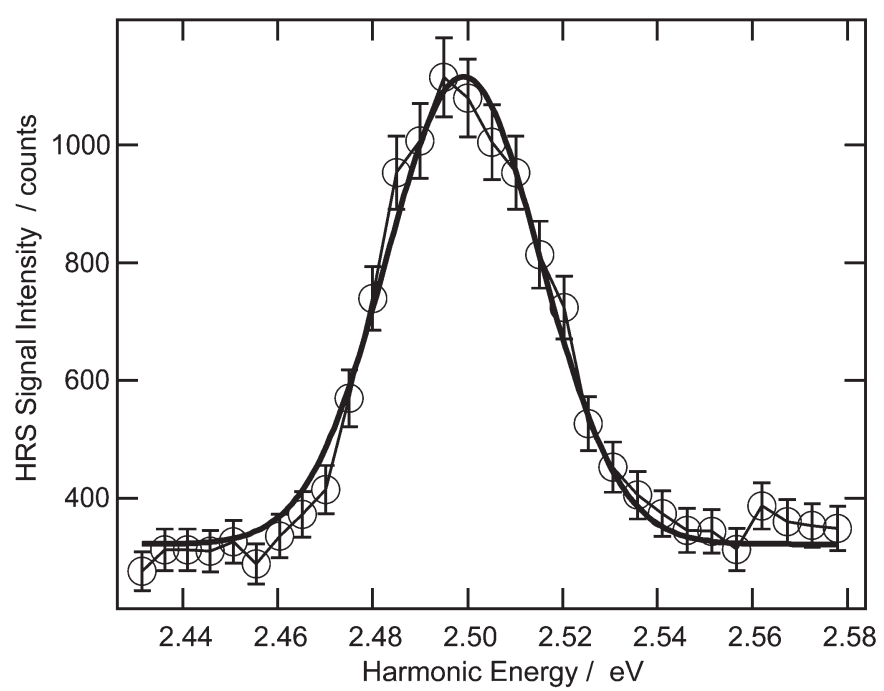

Fig. 2 Monochromaticity of the HRS intensity recorded for a mixed solution of monodispersed gold nanospheres and nanorods with a fundamental energy of $1.25 \mathrm{eV}$ in the $v V$ polarisation configuration. 
spectrum range monitored. The hyper-Rayleigh band has a Gaussian shape and a full width at half maximum of about $5 \mathrm{~nm}$. This width is slightly larger than the expected one considering the width of the spectrum of the incoming femtosecond pulses, indicating that the spectral resolution is limited by the spectrometer. Indeed, because of the rather weak signal recorded, the slits of the spectrometer were opened enough to collect as much light as possible. Experiments were also conducted on pure water and the salt solutions to reject the possibility that the luminescence arises from unreacted reactants or other organic compounds from the particle synthesis like citrate, in the case of the nanosphere synthesis, or other by-products of the reduction reaction. No signal was observed in these measurements although the measurement with the reducing agent and the gold salt simultaneously present in the sample could not be performed since the gold ion reduction into metallic gold nanoparticles is initiated when such a solution is irradiated by the laser. This process is rather efficient for visible incident energies but still active at energies of infra-red photons. ${ }^{19}$ Furthermore, HRS measurements on a $p$ NA solution clearly exhibited the single HRS band without any background signal confirming the origin of the photoluminescence from the nanoparticles sample cell and not from other experimental sources.

Photoluminescence from gold nanoparticles has already been reported in the past although for spherical particles with a smaller diameter of about $5 \mathrm{~nm}$. This luminescence was assumed to vanish for larger particles. ${ }^{20}$ This last assumption is not strictly correct, a strong photoluminescence background being also observed in the monochromaticity of the gold spherical particles. This may stem from the sensitive detection system used to record the HRS light. All these particles, whether nanospheres or nanorods, possess the centrosymmetry property and the HRS intensity collected is very small as compared to the photoluminescence background. The measurement of a photoluminescence background at energies higher than the HRS band energy suggests that the excitation process is at least a three photon process. This yields an energy of excitation of at least $3.75 \mathrm{eV}$, well above the energy for interband transitions from the d-valence band to the sp-conduction band in gold metal. Previous reports have attributed this luminescence background to electron-hole recombinations following multiphoton excitation of bound valence electrons to the conduction band. ${ }^{20}$ This is likely to be the case here although radiative relaxations by electron-hole recombination in metals has a weak probability. ${ }^{21,22}$ It is therefore suggested that the surface of the nanoparticles plays an essential role in favoring radiative relaxation pathways. From the synthesis procedure and surface enhanced Raman spectroscopy studies, it is known that the gold nanospheres are surrounded by a layer of organic compounds, citrate, stabilising agents or other reaction products. ${ }^{23,24}$ For the nanorods, the organic compound is the cation $\mathrm{CTA}^{+}$. Hence surface states are likely to exist and facilitate electron-hole recombination through a radiative process. For nanospheres, the use of ion exchange membranes where citrate is replaced by $\mathrm{SO}_{3}{ }^{-}$have yielded a considerable reduction in the photoluminescence and preliminary investigations on nanoparticles produced in the gas phase and encapsulated within an alumina matrix thin film have clearly demonstrated the absence of such a luminescence background. These particles are free from any surface contaminants due to their preparation in clean vacuum conditions.

The polarisation analysis of the photoluminescence signal from the gold nanorods was performed for different $\gamma \Gamma$ configurations where $\gamma$ stands for the polarisation of the incoming fundamental beam and $\Gamma$ the polarisation of the outgoing light. In all cases, the incoming light was always linearly polarised. The parameters $h$ or $H$ stand for horizontally and $v$ or $V$ for vertically polarised light, 45 standing for the fundamental light polarised at $\pi / 4$ to the vertical or the horizontal axis. In this notation, vertically and horizontally refer to the laboratory coordinates. To perform these measurements, the fundamental energy of the incoming beam was set at $1.19 \mathrm{eV}$ and the monochromator was set at an energy slightly higher than the energy of the HRS light, namely $2.48 \mathrm{eV}$. Irrespective of the polarisation angle $\gamma$ of the incoming fundamental beam, the photoluminescence intensity collected for the vertical and the horizontal polarisation configurations is constant. As a function of the polarisation angle $\gamma$, that is for the input fundamental wave vertically polarised, the photoluminescence is also observed to be unpolarised.

Fluorescence depolarisation experiments have been performed in the past on chromophores in solutions in order to extract rotational diffusion times. ${ }^{25}$ The experimental procedure relies on the irradiation of the sample cell with an incoming beam vertically polarised, and the detection of the outgoing fluorescence at right angle from the irradiation direction through an analyser. In the limit of a fluorescence relaxation time much faster than the rotational diffusion time, the fluorescence is 
vertically polarised. On the opposite, in the limit of a fast rotational diffusion time compared to the fluorescence relaxation time, the fluorescence intensities detected with the analyser set in the vertical and the horizontal position will be identical. Such a behaviour was indeed observed for an aqueous solution of sulforhodamine. In our case, the photoluminescence is not preferentially polarised in one or the other direction, whatever the polarisation of the incoming beam. This conclusion is at first sight surprising since the rotational diffusion time of the nanoparticles is much longer than the expected photoluminescence relaxation time. With a crude single exponential relaxation time adapted for a nanosphere, the rotational diffusion time constant is given by $\tau_{\mathrm{D}}=D / 6$ where $D=k T / 6 V \eta$ is the diffusion coefficient for a sphere of volume $V$ in a medium with a viscosity $\eta$ at a temperature $T{ }^{25}$ For $11 \mathrm{~nm}$ diameter gold particles, $\tau_{\mathrm{D}}$ is about one microsecond, a time much longer than the relaxation time of this luminescence. For nanorods, the relaxation times corresponding to the rotational diffusion time along the short axis and the long axis have a more complicated form but their order of magnitude is similar for comparable axis lengths. The rotational diffusion times along both axes will thus be even longer for the nanospheres and the nanorods of the solution since their size is larger. The complete depolarisation of the photoluminescence signal therefore cannot be attributed to the randomisation of the particles orientation through rotational diffusion motion. Rather, the randomness of the orientation of the different surface organic compounds, gold complexes formed at the surface of the nanoparticles, is responsible for the isotropic luminescence signal. This randomness arises from the almost isotropic orientation of the adsorbed species at the surface of the isotropic particles, provided the nanorods or not too anisotropic, and from the isotropic orientation of the particles in the solution.

\section{Gold nanospheres}

The HRS intensity from a solution of nanoparticles is given by:

$$
I_{\mathrm{HRS}}=G\left\langle N_{\mathrm{w}} \beta_{\mathrm{w}}^{2}+N \beta^{2}\right\rangle I^{2}
$$

where $\beta_{\mathrm{w}}$ and $\beta$ are the respective hyperpolarisabilities of the water molecule and the nanoparticle, and $N_{\mathrm{w}}$ and $N$ their respective numbers per unit volume. $G$ is a constant incorporating all the geometrical factors and $I$ is the fundamental intensity. The expression of eqn. (4) should be corrected for self-absorption but this is unnecessary here since this work is performed at a single photon energy. For gold nanoparticles, the hyperpolarisability was determined to be in the range of $2.0-16.6 \times 10^{-25}$ esu for a particle diameter range of 10-20 nm. ${ }^{3}$ Hence, the expected HRS signals are expected to be a hundred times that of the HRS signal of a bare water solution, the latter signal being within the noise level in our system. Furthermore, the average intensity delivered by the laser source barely reached $100 \mathrm{~mW}$ at the fundamental energy. Hence, HRS signal intensities detected were still very weak and the polarisation analysis of the HRS light for gold nanospheres could not be performed. These signals could undoubtedly be detected but analysis of their polarisation state further decreased their intensities and the statistical errors were too large to be meaningful. The determination of the true dependence of the HRS intensity with these two $\gamma$ and $\Gamma$ angles could not be achieved.

In order to investigate further the experimental data, and compare the expected results for nanospheres with that of nanorods, the theory developed by Dadap et al. may be used to evaluate the expected depolarisation ratio from nanopsheres for the different $\gamma \Gamma$ configurations. ${ }^{6}$ The electric field at the fundamental energy can be written as:

$$
\vec{E}_{0}=E_{0}(\cos \gamma \hat{x}+\sin \gamma \hat{y})
$$

for the fundamental electromagnetic wave at frequency $\omega$ propagating in the $\hat{z}$ direction. The wave is assumed to be linearly polarised with the angle of polarisation $\gamma$ such that for $\gamma=0$ the wave is vertically polarised. Here, unit vectors are written with a hat and we have omitted the time dependence for convenience. Although the origin to the HRS light scattered from the particles is known to be different from an electric dipole origin, one can still use the following expression for the excited electric dipole $\vec{\mu}$ oscillating at the harmonic frequency:

$$
\vec{\mu}=\stackrel{\leftrightarrow}{\beta} \vec{E}_{0} \vec{E}_{0}
$$

Faraday Discuss., 2004, 125, 145-156 
the expression of the hyperpolarisability tensor $\overleftrightarrow{\beta}$ becoming that of an effective one. Without any restrictions on the vanishing and the non vanishing tensor elements of the hyperpolarisability $\overleftrightarrow{\beta}$, and closely following the original work of Bersohn et al. ${ }^{26}$ the three components of the dipole $\vec{\mu}$ are given by:

$$
\mu_{I}=E_{0}^{2}\left[\beta_{I X X} \cos ^{2} \gamma+\beta_{I Y Y} \sin ^{2} \gamma+\left(\beta_{I X Y}+\beta_{I Y X}\right) \cos \gamma \sin \gamma\right]
$$

where the subscript $I$ stands for either $X, Y$ or $Z$, the three laboratory coordinates. For a second harmonic process with a degenerate fundamental field, the condition $\beta_{I X Y}=\beta_{I Y X}$ has to be fullfilled. The expression of eqn. (7) can then be compared with the theoretical expression given by Dadap et al. ${ }^{6}$ Indeed, from the theory it turns out that $\vec{\mu}$ is given by:

$$
\vec{\mu}=\vec{p}-\frac{i K_{1}}{6} \vec{Q}(\hat{n})
$$

where the electric dipole $\vec{p}$ is nonlocally excited and the electric quadrupole $\vec{Q}(\hat{n})$ is an effective quadrupole locally excited, to take the terminology used in that theoretical work. $K_{1}$ is the harmonic wave vector modulus in the outer medium outside the particle. The electric dipole and electric quadrupole can be written in the laboratory frame $(\hat{x}, \hat{y}, \hat{z})$, yielding:

$$
\begin{gathered}
\vec{p}=a \hat{\kappa}=a \hat{z} \\
\vec{Q}(\hat{n})=b \sin \gamma(\cos \gamma \hat{x}+\sin \gamma \hat{y})
\end{gathered}
$$

if the direction of observation $\hat{n}$ is set equal to the $\hat{y}$ direction. The unit vector $\hat{\kappa}$ is the unit vector supporting the direction of propagation, the direction of the wave vector $\vec{k}$, therefore $\hat{\kappa}=\hat{z}$. The parameters $a$ and $b$ are complex parameters scaling with the volume of the nanoparticle and incorporating linear combinations of the three microscopic non vanishing surface susceptibility elements. Direct identification of eqn. (7) with eqns. (8)-(9) yields:

$$
\begin{aligned}
& b=\beta_{X X Y}+\beta_{X Y X}, \beta_{X X X}=\beta_{X Y Y}=0 \\
& a=\beta_{Z X X}=\beta_{Z Y Y}, \beta_{Z X Y}=\beta_{Z Y X}=0
\end{aligned}
$$

Other relations may also be determined for the elements of the form $\beta_{Y J K}$ but are unnecessary for the remainder of the derivations. The electric field scattered at the harmonic frequency is detected in the $\hat{y}$ direction, that is, at right angles to the fundamental beam propagation, thus its expression is given by:

$$
\vec{E}(2 \omega)=C(\hat{y} \times \vec{\mu}) \times \hat{y}
$$

and thus the HRS intensity at the harmonic frequency $2 \omega$ retains the general form:

$$
I(2 \omega)=K\left[\left|\mu_{X}\right|^{2}+\left|\mu_{Z}\right|^{2}\right] I^{2}
$$

where $C$ and $K$ are pure constants at a given fundamental frequency. Since this work focusses on the measurement of the HRS signal intensity for different output polarisation angles $\Gamma$, eqn. (12) is rewritten as a set of two equations for the two HRS polarisation configurations, respectively:

$$
\begin{gathered}
I^{V}(2 \omega)=K\left|\beta_{X X Y}\right|^{2} \sin ^{2} 2 \gamma I^{2} \\
I^{H}(2 \omega)=K\left|\beta_{Z X X}\right|^{2} I^{2}
\end{gathered}
$$

for the vertically and the horizontally polarised light. Thus, it appears that the horizontally polarised HRS light is independent of the angle of polarisation of the incoming light at the fundamental frequency whereas the vertically polarised HRS light is a bell-shaped curve with the angle of polarisation $\gamma$ of the fundamental wave. Also, it appears that the vertically polarised light is of pure electric quadrupole origin whereas the horizontally polarised light is of pure electric dipole origin. This experimental configuration is therefore adequate to separate these two contributions. The expected dependence suggests that the HRS signal will be vanishing for $\gamma=v, h$ for the analyser set to collect the $V$ polarisation and constant irrespective of the angle of polarisation $\gamma$ for the analyser set in the position $H$. 
A closer look at the source to the two elements of the hyperpolarisability tensor $\overleftrightarrow{\beta}$ indicates that the element $\beta_{Z X X}$ has its origin in the nonlocally excited electric dipole $\vec{p}$ whereas the element $\beta_{X X Y}$ has its origin in the locally excited electric quadrupole $\vec{Q}(\hat{n})$. Experiments aiming at determining the absolute value of the hyperpolarisability for gold nanoparticles as a function of the fundamental energy have shown that for particles with $11 \mathrm{~nm}$ diameter, it was not possible to detect the electric quadrupole contribution, ${ }^{8}$ as opposed to the work performed on the larger $32 \mathrm{~nm}$ diameter silver nanoparticles by Hao et al. ${ }^{7}$ Two reasons explain these results. The first reason is the larger size of the particles used by Hao et al., thus enhancing the weight of the electric quadrupole since the weight of higher multipoles rises as the ratio $a / \lambda$ increases due to retardation effects. The second reason is due to the metal used and the larger imaginary part of the gold dielectric function at the harmonic energy around $2.50 \mathrm{eV}$ damping the SP resonance effect. The quadrupolar contribution is expected to be enhanced when the condition $3 \varepsilon(2 \omega)+2=0$ is fullfilled whereas the dipolar contribution is enhanced for the usual condition $\varepsilon(2 \omega)+2=0$. In these two conditions, $\varepsilon(2 \omega)$ is the dielectric constant of the metal at the harmonic frequency. In this work, the fundamental frequency was set around 1.15-1.25 eV, therefore the harmonic frequency at $2.30-2.5 \mathrm{eV}$ was close to the resonance frequency of the dipolar SP excitation of the gold nanospheres in water. The quadrupolar SP resonance appears at a higher energy and was not excited. The element $\beta_{X X Y}$ of the hyperpolarisability is therefore expected to vanish, or to be rather small. No signal should thus be recorded for the polarisation angle set at $\Gamma=0$. These observations clearly call for improvements in the experimental apparatus to unambiguously confirm these data. This work is currently under progress in our laboratory.

\section{Gold nanorods}

In a previous work, pyridine was introduced into the sample cell containing the aqueous solution of gold spherical nanoparticles and aggregation of the particles into large aggregates was thus initiated. ${ }^{11}$ The mechanism for this aggregation is the substitution of the stabilising agent, namely citrate which is present in excess in the synthesis, by pyridine which adsorbs strongly at the surface of gold metal. The electrostatic stabilisation of the solution is thus broken since pyridine is neutral whereas citrate is negatively charged. At the initial stages of the aggregation process, it is known that linear aggregates of a few nanoparticles are produced, as demonstrated by TEM images. ${ }^{12}$ These images also show that the particles retain their character and therefore do not fuse together into larger particles. This latter process can nonetheless be started under laser irradiation with adequate photon energies, a regime not attained in this work. In the early regime of aggregation, when aggregates retain a linear geometry, it has been reported that the HRS signal increases by a factor of about ten before saturating when the aggregates start to form complex geometries with a radius of gyration close to the wavelength of light. ${ }^{11}$ This regime can be successfully modeled with ellipsoid particles of different aspect ratio and the proper account of the field factors at the fundamental and the harmonic energies in the source to the HRS intensity enhancement correctly describes the experimental data. For larger aggregates, the model breaks down because retardation effects must be properly taken into account. In order to investigate further the origin of the HRS intensity from these linear aggregates, it was desired to investigate nanorods and compare the results with the data obtained for linear aggregates of spherical particles.

The absolute HRS intensity from the solution of nanorods and nanospheres was clearly much larger than the one recorded for the solution of spherical particles only. With the fundamental photon energy at $1.25 \mathrm{eV}$, the harmonic photon energy is slightly off resonance with the transverse SP resonance and thus the HRS intensity is resonantly enhanced through the SP resonance, a condition yielding large intensities. The HRS intensity reported on Fig. 2 is about twenty times that obtained for a $0.1 \mathrm{M} p \mathrm{NA}$ in methanol, once the photoluminescence background is removed. This nanorod HRS signal intensity is thus also SP resonantly enhanced. It was reported above that nearly three quarters of the solution is made from nanopsheres. In fact, it seems that the HRS response from the nanospheres is negligible. Indeed, because of the tight focussing of the fundamental incident beam, it is possible to convert the nanorods into nanopsheres. ${ }^{27}$ This was clearly observed by recording UV-visible absorption spectra after long irradiation times in such conditions. The HRS signal intensities obtained from these solutions rich in nanospheres were gradually disappearing, supporting the fact that the HRS intensity of this mixed solution do indeed 

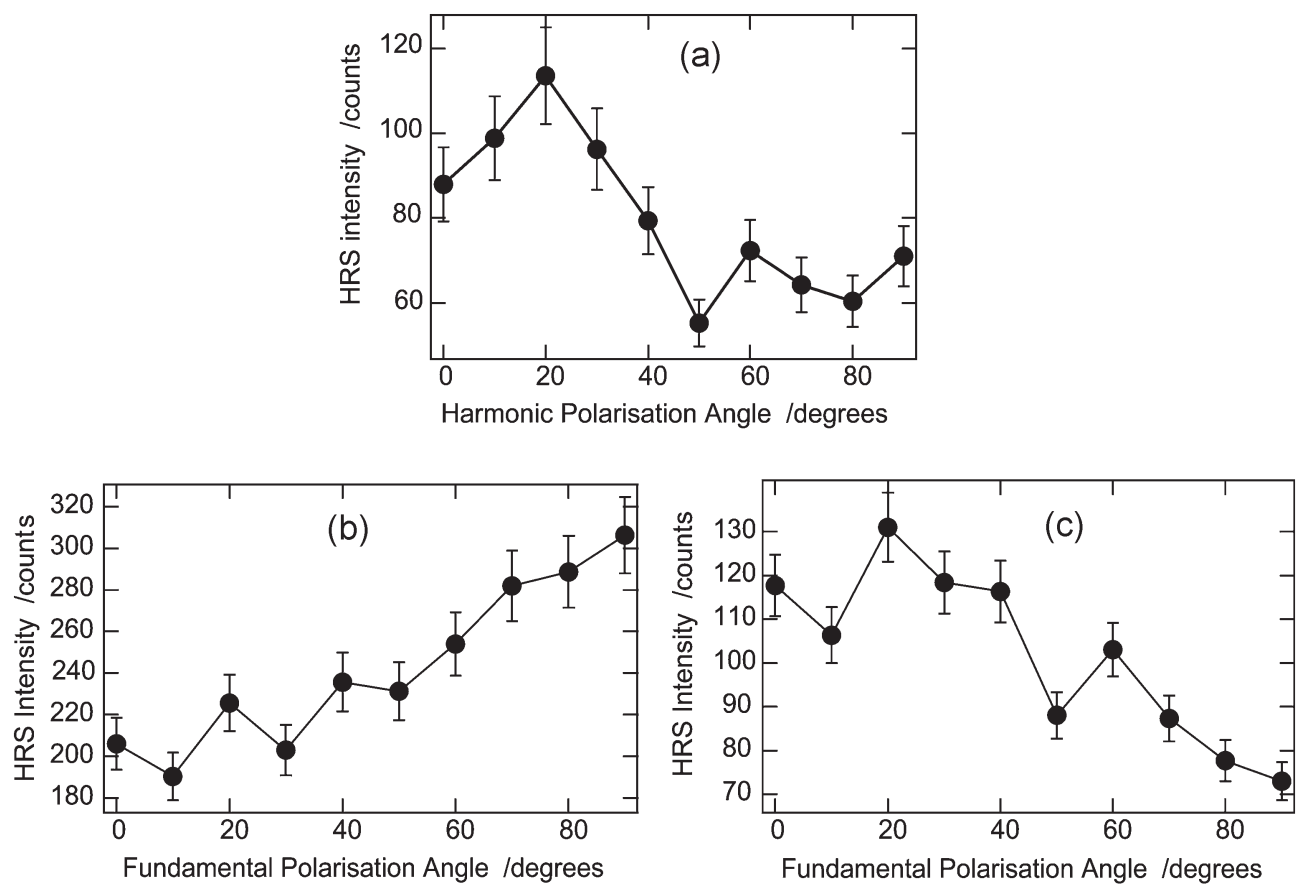

Fig. 3 Polarisation dependence of the HRS intensity for gold nanorods recorded with a fundamental energy of $1.21 \mathrm{eV}$, (a) for the $v \Gamma$ configuration, (b) for the $\gamma H$ configuration and (c) for the $\gamma V$ configuration.

arise from the nanorods. This result suggests that the hyperpolarisability of nanorods is much larger than that of nanospheres. Nevertheless, a comparison with the absolute hyperpolarisability value of nanospheres cannot be obtained straightforwardly. Indeed, owing to the centrifugation process and the presence of a mixture of nanorods and nanospheres in the solution, the exact number density of nanorods is unknown. Also present in the solution is a distribution of nanorods with different aspect ratios. Besides this problem, the HRS response from the nanospheres may still contribute to the overall signal and the HRS signal intensities must therefore be corrected for this contribution. Finally, the long axis of the nanorod being no longer negligible in front of the fundamental wavelength, coherent contributions cannot be ruled out. Further work is under way to determine this absolute hyperpolarisability value for the nanorods.

In the previous work on linear aggregates, the large increase of the HRS response from a nanorod solution was interpreted in terms of the local field factors $f(\omega)$ for ellipsoidal nanoparticles of gold at both the fundamental and the harmonic energies:

$$
f(\omega)=\frac{4 \pi}{V} \sum_{i} w_{i} \frac{\operatorname{Im}\left(\alpha_{1}^{i}(\omega)+2 \alpha_{\mathrm{t}}^{i}(\omega)\right)}{\operatorname{Im} \varepsilon(\omega)}
$$

here $\alpha_{1}^{i}(\omega)$ and $\alpha_{t}^{i}(\omega)$ are the longitudinal and transverse polarisability of the ellipsoids. In eqn. (14), $\varepsilon(\omega)$ is the dielectric constant of gold with respect to that of water and the $w_{i}$ coefficients are the different weights of the ellipsoid aspect ratio distribution since it is not expected to have a particularly sharp distribution around the average aspect ratio. The agreement between this simple model of the HRS response from ellipsoids and the experimental data reported for linear aggregates of spherical particles underlines the strong coupling between the nanoparticles in the aggregate. The coupling is achieved through induced-dipole-induced-dipole interactions, a coupling the strength of which is known to fall rapidly when the particles are taken apart. Thus, such a strong coupling requires that the spheres are in close contact. The model of local field factors of ellipsoids is thus expected to correctly account for the HRS intensities of linear aggregates and 
nanorods. The synthesis of nanorods of adjustable aspect ratio further allow the study of linear assemblies with larger long axes as compared to the geometries obtained through the aggregation process. From these consideration, through comparison between an aggregating solution and a solution of nanorods, the HRS intensity should prove useful in determining the onset of the deviation from linear aggregates.

The polarisation analysis of the HRS intensity of the nanorods is reported on Fig. 3(a)-(c) for different polarisation configurations $\gamma \Gamma$. A clear depolarisation ratio of the HRS intensity is observed for the given $\gamma=v$ polarisation of the incoming fundamental light, the analyser being rotated from the $V$ to the $H$ position. This curve also suggests that the HRS light is elliptically polarised since no position of the analyser can extinguish the HRS light. These curves are clearly different from the behaviour expected for nanospheres and therefore should prove useful to monitor the aggregation process in situ in real time. Furthermore, the data suggest that the HRS response arises principally from the nanorods and not the nanospheres since the polarisation curves are clearly different from the expected curves for spheres. Nanorods also present a center of inversion and therefore the origin of the HRS intensity collected from the nanorods is not arising from an electric dipole contribution. However, the model as derived above for the nanospheres cannot be applied for nanorods since they have their own reference frame attached to their anisotropy, a reference frame different from the laboratory frame. In the case of the nanospheres, the two reference frames were identical because the incident light polarisation state was defining the reference frame. Thus averaging over all the possible orientations of the nanorods in the solution must be accounted for. Nonetheless, since the incident photon energy is fixed at $1.2 \mathrm{eV}$, the HRS signal cannot be SP enhanced through the longitudinal SP mode because it is lying at a too low harmonic energy. The exact polarisation state of the HRS light is thus not straightforward to determine but this will be reported in a future work. Considering the size of the nanorods, it is though expected that multipolar radiation at the harmonic energy of the excited dipole, and possibly higher multipoles, will have to be taken into account further complicating the expected expressions for the HRS intensity from nanorods.

\section{Conclusion}

The analysis of the HRS intensity collected from a solution of a mixture of gold nanorods and nanospheres confirms that the HRS signal essentially arises from the nanorods. The HRS intensity is also SP resonantly enhanced, like that of gold nanospheres, through the excitation of the SP transverse mode. The HRS response from the nanorods is in agreement with the model derived for linear aggregates of spherical nanoparticles. In that model of linear aggregates, a strong electromagnetic coupling between the particles because of their close contact was assumed. Further improvments must be developed though to take into account multipolar radiation and possible coherent radiation from nanorods with long axes no longer much smaller than the fundamental wavelength. Polarisation analysis of the HRS intensity collected from the solution of nanospheres could not be determined owing to the weaknesses of the signal. On the opposite, the polarisation analysis of the HRS intensity from gold nanorods has been performed and exhibit a clear depolarisation ratio. The model available cannot be straightfowardly applied to nanorods and therefore further work must be pursued in this direction. In a similar way, it is found that the nanorod solution contains a large amount of nanospheres and this observation precludes the absolute determination of the hyperpolarisability of the nanorods.

The careful analysis of the HRS intensities collected have also shown a strong background of photoluminescence. This luminescence is attributed to the radiative electron-hole recombination following a multiphoton excitation of d-valence electrons into the sp-conduction band. The radiative relaxation channel, normally with a vanishing probability, is probably favored by surface states arising from metal-organic complexes at the surface of the nanoparticles.

\section{References}

1 U. Kreibig, M. Vollmer, Optical Properties of Metal Clusters, Springer-Verlag, Berlin, 1995.

2 K. Clays, E. Hendrickx, M. Triest and A. Persoons, J. Mol. Liq., 1995, 67, 133.

3 P. Galletto, P. F. Brevet, H. H. Girault, R. Antoine and M. Broyer, Chem. Commun., 1999, 581.

Faraday Discuss., 2004, 125, 145-156 
4 F. W. Vance, B. I. Lemon and J. T. Hupp, J. Phys. Chem. B, 1998, 102, 10091.

5 G. S. Agarwal and S. S. Jha, Solid State Commun., 1982, 41, 499.

6 J. I. Dadap, J. Shan, K. B. Eisenthal and T. F. Heinz, Phys. Rev. Lett., 1999, 83, 4045.

7 E. C. Hao, G. C. Schatz, R. C. Johnson and J. T. Hupp, J. Chem. Phys., 2002, 117, 5963.

8 I. Russier-Antoine, J. Nappa, E. Benichou, Ch. Jonin and P. F. Brevet, to be published.

9 A. K. Sarychev and V. M. Shalaev, Phys. Rep., 2000, 335, 275.

10 F. Remacle, C. P. Collier, J. R. Heath and R. D. Levine, Chem. Phys. Lett., 1998, 291, 453.

11 P. Galletto, P. F. Brevet, H. H. Girault, R. Antoine and M. Broyer, J. Phys. Chem. B, 1999, $103,8706$.

12 C. G. Blatchford, J. R. Campbell and J. A. Creighton, Surf. Sci., 1982, 120, 435.

13 D. A. Weitz and M. Oliveria, Phys. Rev. Lett., 1984, 52, 1433.

14 N. R. Jana, L. Gearheart and C. J. Murphy, Langmuir, 2001, 17, 6782.

15 J. Turkevich, P. C. Stevenson and J. Hillier, Discuss. Faraday Soc., 1951, 11, 55.

16 S. Link and M. A. El-Sayed, J. Phys. Chem. B, 1999, 103, 8410.

17 S. Link, M. B. Mohammed and M. A. El-Sayed, J. Phys. Chem. B, 1999, 103, 3077.

18 P. B. Johnson and R. W. Christy, Phys. Rev. B, 1972, 3, 4370.

19 J. P. Abid, A. W. Wark, P. F. Brevet and H. H. Girault, Chem. Commun., 2002, 792.

20 J. P. Wilcoxon, J. E. Martin, F. Parsapour, B. Wiedenmann and D. F. Kelley, J. Chem. Phys., 1998, 108, 9137.

21 G. T. Boyd, Z. H. Yu and Y. R. Shen, Phys. Rev. B, 1986, 33, 7923.

22 C. K. Shen, A. R. B. de Castro and Y. R. Shen, Phys. Rev. Lett., 1981, 46, 145.

23 M. A. Hayats, Colloidal Gold: Principles, Methods and Applications, Academic Press, New York, 1989, vol. 1.

24 M. Kerker, O. Siiman, L. A. Bumm and D. S. Wang, Appl. Optics, 1980, 19, 3253.

25 G. R. Fleming, J. M. Morris and G. W. Robinson, Chem. Phys., 1976, 17, 91.

26 R. Bersohn, Y. H. Pao and H. L. Frisch, J. Chem. Phys., 1966, 45, 3184.

27 S. Link, C. Burda, M. B. Mohamed, B. Nikoobakht and M. A. El-Sayed, J. Phys. Chem. A, 1999, 103, 1165. 